Copyright (C) 2019 University of Bucharest

Printed in Romania. All rights reserved

ISSN print: $1224-5984$

ISSN online: $2248-3942$
Rom Biotechnol Lett. 2019; 24(6): 961-965

doi: $10.25083 / \mathrm{rbl} / 24.6 / 961.965$

Received for publication, December, 18, 2018

Accepted, July, 4, 2019

Original paper

\title{
Anesthesia protocols and techniques for imaging diagnosis procedures in small animals
}

\author{
ANDRA DEGAN ${ }^{1}$, ALEXANDRU ŞONEA ${ }^{1}$ \\ University of Agronomic Science and Veterinary Medicine, ${ }^{1}$ Faculty of Veterinary Medicine \\ Bucharest, 59 Marasti Blvd, District 1, Bucharest, Romania
}

\begin{abstract}
Nowadays, imaging diagnosis play an important role in veterinary medicine, magnetic resonance imaging (MRI) being considered the top tool in diagnosing pathologies of different tissues and organs. Same as in pediatric medicine, this procedure has an impediment, represented by general anesthesia, whose effects upon the organism can increase risks. The importance of anesthesia during imaging diagnosis stands in the fact that the patients have to be motionless in order to achieve good quality images. General anesthesia for small animals during these procedures can be done with either the use of a total intravenous anesthesia or by using inhalation anesthesia. Both techniques described in studies have provided good results and are intended in helping practitioners all over the world.

The purpose of this article is to highlight the most common anesthetic protocols and risks associated with them, as well as the aspects most seen by an anesthetist and imagist during an MRI scan.
\end{abstract}

Keywords Anesthesia, animals, magnetic resonance imaging.

To cite this article: DEGAN A, ȘONEA A. Anesthesia protocols and techniques for imaging diagnosis procedures in small animals. Rom Biotechnol Lett. 2019; 24(6): 961-965. DOI: $10.25083 / \mathrm{rbl} / 24.6 / 961.965$

*Corresponding author: ANDRA DEGAN, Faculty of Veterinary Medicine Bucharest, 59 Marasti Blvd, District 1, Bucharest, Romania

E-mail: andra.degan@yahoo.com 


\section{Introduction}

Anesthesia is often required for patients undergoing diagnostic procedures that utilize dangerous devices or whenever the animals are not co-operative or are undergoing painful procedures.

The use of anesthesia also facilitates positioning the animals in correct position for the scan (Marcus, A., 2009; Toby T. et al, 2018). Moreover, it is important to achieve these effects in order to reduce the stress and anxiety of the patients as well as to obtain good quality images for a proper diagnosis (Leppänen, M. K. et al, 2006).

Magnetic resonance imaging (MRI) utilizes a powerful static magnetic field, the main component of it being the magnet. MRI is a procedure which is widely used in the veterinary medicine as a modality for diagnostic imaging (Stefania Scarabelli et al, 2016). At this moment in time, it is considered to be the most versatile technique that is able to identify the anatomical, functional and physiological features of different tissues and organs (Driehuys B. et al, 2008). The high accuracy of this technique allows for a better diagnostic of different neurological, musculoscheleal and also oncological pathologies (Marian Soare \& Constantin Vlăgioiu, 2012).

MRI also provides the advantage of nonionizing 3D imaging as well as the benefit of its noninvasive quality, meaning that animals are not exposed to radiations. The difference between human and animal MRI protocols is that animals have to be anesthetized meaning the exposure to risk is then associated with the general anesthesia (Jordi L. Tremoleda et al, 2018). In human medicine, anesthesia has been used for children during the MRI scan, with different anesthetic protocols being able to immobilize the patients and at the same time erase anxiety, thus ensuring a satisfactory outcome of the scan (Mahmound Reza M., et al, 2017). It has been shown that noise reduction can also allow lighter planes of anesthesia in children (Oğurlu, M. et al, 2012).

\section{Pre-anesthetic management and anesthesia protocols in animals under- going imaging diagnosis procedures}

Before any sedation or general anesthesia, it is important to follow a set of preparatory steps. The preanesthetic evaluation's purpose is to provide any information related to patient's status, such as: individual risk factors, history, physical examination, laboratory and cardiac exams. Once the patient belongs to an ASA group risk (ASA Physical Status Classification System), the anesthetist can then create an individualized anesthetic plan (Richard B. et al, 2011).

Premedication consists in the administration of tranquilizers, sedatives, opioids or combination of these drugs. All these drugs help in restraining the animal, reducing its stress and lowering the dose of induction and inhalant anesthetic used. Choosing the most suitable medication for the patient can be a challenging task. The decision has to be taken according not only to patient's health status but also to patient's temperament and type, the duration of the procedure and personal preference (Bednarski, R. M. et al, 2015).

Induction of anesthesia can be performed with the use of propofol and alfaxalone or a combination of ketaminemidazolam, ketamine-propofol. Studies have shown that propofol and alfaxalone are drugs that allow for an appropriate intubation while also offering the patient a good and rapid recovery (Alessandra Mathis et al., 2012; Maria Amengua et al, 2013).

General anesthesia is typically maintained with inhalant anesthetics, like isoflurane or sevoflurane, although it can be achieved with the use of injectable drugs, in repeated boluses or as a continuous infusion (Richard B. et al, 2011). During maintenance of general anesthesia, fluidotherapy has to be performed in all patients, usually with the use of crystalloids at a rate of $3-5 \mathrm{ml} / \mathrm{kg} /$ hour.

Recovery has the same importance as any other step of a general anesthesia due to the fact that complications can be seen throughout this period.

Magnetic resonance imaging is a diagnostic tool widely used in veterinary species where a contrast study is often required for diagnosis. MRI remains the most versatile imaging modality used for anatomical, functional and physiological characterizations of tissues and organs (Driehuys B. et al, 2008). One of the challenges associated with MRI in animals is considered to be the need of general anesthesia which can alter more or less the organism and different body functions (Tremolda L. et al, 2018).

Injectable and inhaled anesthetics are both used in imaging diagnosis procedure. However, inhalational anesthesia is the recommended method as it provides a more rapid induction and recovery. The use of injectable agents often causes a prolonged recovery time compared to inhalant agents and, in order to achieve a steadier plasma concentration, some injectable drugs are used via infusion (Lovell DP, 1986; Tremolda L. et al, 2018). The first step in performing general anesthesia is the premedication period. The purpose of premedication is to ensure the loss of anxiety in the patient and to smooth the recovery from anesthesia (Dugdale, 2010). The administration of a combination between an opioid and a benzodiazepine or a dissociative agent, in premedication has reduced the dose of required propofol for intubation or during general anesthesia for MRI, compared to the use of an opioid alone (Degan A. et al, 2018).

There have been various studies performed regarding the effects of different anesthetic protocols on different periods of general anesthesia. Compared to other diagnosis imaging techniques, MRI strictly forbids the use of any tools or equipment containing metal during the scanning procedure. For this reason, if the anesthetic machine is not fitted with compatible equipment, monitoring the patient can prove to be very challenging (Julie A. Smith, 2015). During the last few years, premedication of patients undergoing an MRI scan has involved the use of anesthetics belonging to different classes of drugs, most commonly with 
a combination of an opioid and a tranquilizer. The majority of available studies show propofol or alfaxalone being used as induction agents before the maintenance period (Lozano A.J. et al, 2009; Carolina P. Jimenez et al, 2012). In one study, the authors used meperidine in a dose of $3 \mathrm{mg} / \mathrm{kg} \mathrm{IM}$ as premedication and propofol IV for induction. Dogs were allocated to three different groups, depending on the volatile anesthetic (Isoflurane, Sevoflurane, Desflurane) used. The results have shown that all three anesthetics are suitable for use in dogs. Recovery times were significantly faster for the patients in receiving desflurane than the ones receiving isoflurane, for both extubation and sternal recumbency (Lozano A.J. et al, 2009). A rapid but calm recovery is desirable especially in patients with neurological disease, in order to avoid excitement and an increase in intra-cranial pressure. The premedication and also the recovery time are important factors when keeping in mind the wellbeing of the patient (Scheller et al, 1990; Clarke, 1999). The importance of good quality sedation was also demonstrated by Jimenez C et al. (2012), where patients that were more sedated in the preoperative period recovered better than the less sedated ones. Besides the degree of sedation, the recovery can be influenced by many factors, like the drugs used and animal's behavior. In the case of horses, the same results were obtained after the use of desflurane compared to isoflurane (Bradbrook et al, 2006). Compared to animals, the use of sevoflurane in children during the maintenance phase of general anesthesia can increase the restlessness in the postoperative period, compared with propofol or halothane (Cravero et al, 2000; Picard et al., 2000). Both propofol and alfaxalone are short acting hypnotic agents with a rapid onset of actions, used in the induction of anesthesia. One study focused of a group of 42 dogs which were premedicated with methadone $(0.2 \mathrm{mg} / \mathrm{kg}$ IM or $0.1 \mathrm{mg} / \mathrm{kg} \mathrm{IV})$ and induced with propofol or alfaxalone. As the purpose of this study was to assess the recovery quality, the results obtained by the time the animal was able to maintain their sternal recumbency, demonstrated that dogs receiving alfaxalone recovered less well than those sedated with propofol. Even so, the quality of the recovery in all animals was generally good (Jimenez C. et al, 2012).

Another technique of performing and maintaining a general anesthesia is the use of a total intravenous anesthesia (TIVA), instead of inhalation anesthesia. In pediatric medicine, this technique is well studied and described. Propofol, usually used as an induction agent can also be administered in continuous infusion in order to maintain anesthesia. A TIVA of propofol was used in children where the protocol seemed to have an effective sedative effect, especially when a low dose of dexmedetomidine $(0.5 \mathrm{mcg} / \mathrm{kg})$ was added. The difference in a group of children that had an addition of dexmedetomidine compared to those receiving only propofol was statistically significantly ( $p$ value<0.0001) (Nagoshi M. et al, 2018). A good preservation of upper airway patency and a rapid recovery was obtained after the administration of a propofol TIVA in a study conducted on children patients undergoing an MRI procedure (Usher AG. et al. 2004).
In a study involving dogs suffering from intercranial disease, the patients were prepared for an MRI by either receiving propofol in CRI or isoflurane. None of the patients were premedicated and all were induced and intubated with the use of propofol IV to effect. Patients maintained with propofol had higher arterial pressure, decreased regimens for dopamine and better recoveries compared to those who had received isoflurane (Caines D. et al, 2014).

In order to achieve a good quality induction or even a better recovery from anesthesia, anesthetists use different protocols which include co-induction agents like ketamine or benzodiazepines (Martinez T.F. \& Leece E.A, 2014). During the induction period, the use of midazolam in combination with either propofol or alfaxalone for patients undergoing an MRI or CT procedure, was evaluated. Dogs were premedicated with Fentanyl IV and the anesthesia was maintained with a TIVA of propofol or alfaxalone. Induction was made either with propofol and saline, propofol with midazolam, alfaxalone with saline or alfaxalone with midazolam. The results of the study showed a decrease in the induction doses of propofol and alfaxalone, as well as an improvement in the quality of induction and a decrease in the rate of propofol used for TIVA (Liao P. et al, 2017).

\section{Monitoring and complications during general anesthesia for imaging diagnosis}

Due to the fact that all anesthetic procedures are related to complications and possible death, it is important to make a proper evaluation of the patient and assess the anesthetic risk so as to minimize the unpleasant changes. Hence the physical health status assessment is an important preanesthetic consideration. Most of the anesthetic agents cause cardiopulmonary depression and neurological depression. (Hall LW et al, 2001). Using inhalant anesthesia the risks for an emergency situation are decreased but not eliminated. The most common problems expected are hypoxemia, hypoventilation, hypotension, cardiac arrhythmias, acidosis and hypothermia (Costea R., 2016). Hyperthermia is less common compared to hypothermia, hypercapnia and hypocapnia are complications also seen, in which case the ventilator settings have to be adjusted so the end-tidal carbon dioxide $\mathrm{EtCO}_{2}$ is between $35-45 \mathrm{mmHg}$.

Monitoring of the patients during every general anesthesia is as important as any other step of anesthesia. Considering that most monitoring equipment and accessories are not designed for use in the MRI environment, monitoring the patients can be more difficult and in some cases the anesthetist has to work with less tools which can lead to increased risks (Julie A.S., 2015).

Hypothermia is a concern as MRI rooms can be very cold. At the present time, there are no commercially available electrical warming systems designed for use in an MRI room. Hot water blankets or simple blankets can be used, but it might be necessary to remove them depending on the region scanned (Julie A.S., 2015). Even the use of a heat and moisture exchanger (HME) cannot help in 
preventing heat loss and preserving body temperature during MRI (Khenissi L. et al, 2017). HME is a device placed between the patient and the breathing system and has the aim to conserve exhaled water and return it to the patient in the inspired gas (Dorsch \& Dorsch 2014). For a study involving dogs, patients weighing less than $10 \mathrm{~kg}$ (Khenissi L. et al, 2017) were premedicated with an opioid (butorphanol) and dexmedetomidine, induced with propofol and maintained with isoflurane in $100 \%$ oxygen during the entire MRI scan. Whilst the use of an HME had no benefit in preserving body temperature, the patients premedicated with dexmedetomidine maintained better rectal temperature compared to dogs that were administered other drugs. Unfortunately, hypothermia can affect the cardiovascular system, producing arrhythmias, hypotension and bradycardia. It also bares consequences on the respiratory system and can decrease metabolic rate (Amstrong et al, 2005).

Some breeds, like Dachshunds might experience lower heart rates much more frequent than others, probably due to a lower rectal temperature during general anesthesia. Therefore, breeds with fine-hair coating can be expected to require anticholinergic drugs and warming during MRI (Rebecca Harrison et al, 2012).

One of the most common complications seen during general anesthesia is hypotension which in most of the cases can be corrected by reducing the inhalant anesthetic, increasing fluid rate and using inotrope and vasopressor drugs. The purpose of fluidotherapy is to maintain the homeostasis, loss covering or fluid resuscitation. Colloids were not used frequently compared to crystalloids. Greater fluid volumes are required to meet the same targets with crystalloids than with colloids with an estimated ratio of 2.77. There is no evidence from randomized controlled trials that resuscitation with colloids reduces the risk of death, compared to resuscitation with crystalloids (Costea Ruxandra et al, 2017). A 7 year old Bull Terrier underwent an MRI procedure which unfortunately was aborted due to severe hypotension. Patient was premedicated with acepromazine and meperidine, induced with ketamine and propofol and maintained with isoflurane in oxygen. Despite the fact that all the measures above where taken in order to combat hypotension, they returned no beneficial result. Furthermore, the administration of dopamine aggravated the hypotension (Carvalho, E. R et al, 2018).

Besides all these side effects that general anesthesia has on the main functions of the body, has also been demonstrated it plays an important role in decreasing tear production. Therefore an adequate supply of tears covering the partially exposed anterior segment of the globe and the associated adnexa is necessary for optical integrity, maintenance of the cornea and normal eye function. The combination of Midazolam and Butorphanol in premedication, followed by Propofol and maintenance with Isoflurane is a commonly used and appropriate anesthetic protocol for geriatric dogs, this drug combination reduces aqueous tear production as determined by use of the STT-Shirmer test (Tudor R. et al, 2018).

\section{References}

1. AMENGUAL, M., FLAHERTY, D., AUCKBURALLY, A., BELL, A.M., SCOTT, E.M., \& PAWSON, P. (2013). An evaluation of anaesthetic induction in healthy dogs using rapid intravenous injection of propofol or alfaxalone. Veterinary anaesthesia and analgesia, 40(2), 115-123.

2. ARMSTRONG, S.R., ROBERTS, B.K., \& ARONSOHN, M. (2005). Perioperative hypothermia. Journal of Veterinary Emergency and Critical Care, 15(1), 32-37.

3. BEDNARSKI, R.M. (2015). Dogs and cats. Veterinary anesthesia and analgesia: the fifth edition of Lumb and Jones, 817-826.

4. BEDNARSKI, R., GRIMM, K., HARVEY, R., LUKASIK, V.M., PENN, W. S., SARGENT, B., \& SPELTS, K. (2011). AAHA anesthesia guidelines for dogs and cats. Journal of the American Animal Hospital Association, 47(6), 377-385.

5. BRADBROOK C, SIGURDSSON S, BORER K et al (2006). A comparison in clinical cases of recovery from anaesthesia following maintenance with desflurane or isoflurane in horses: preliminary results. Proceedings of the WCVA congress, Santos, Brazil (abstract).

6. CAINES, D., SINCLAIR, M., VALVERDE, A., DYSON, D., GAITERO, L., \& WOOD, D. (2014). Comparison of isoflurane and propofol for maintenance of anesthesia in dogs with intracranial disease undergoing magnetic resonance imaging. Veterinary anaesthesia and analgesia, 41(5), 468-479.

7. CARVALHO, E.R., GAROFALO, N.A., GIROTTO, C.H., \& NETO, F.J.T. (2018). Hypotension Aggravated by Dopamine in a Dog Under Isoflurane Anesthesia. Acta Scientiae Veterinariae, 46(1), 246.

8. CLARKE, K.W. (1999). Desflurane and sevoflurane: new volatile anesthetic agents. Veterinary clinics of North America: Small animal practice, 29(3), 793-810.

9. COSTEA, R. (2016). Anesthesia considerations for critically ill patients.

10. COSTEA, R., DEGAN, A., \& TUDOR, R. (2017). Crystalloids/colloids ratio for fluid resuscitation during anesthesia. Scientific Works. Series C. Veterinary Medicine, 63(1), 65-66.

11. CRAVERO, J., SURGENOR, S., \& WHALEN, K. (2000). Emergence agitation in paediatric patients after sevoflurane anaesthesia and no surgery: a comparison with halothane. Pediatric Anesthesia, 10(4), 419-424.

12. DEGAN, A.I., TUDOR, R.G., IVASCU, C., COSTEA, R., \& SONEA, A. (2018). The influence of different anesthetic protocols on the use of propofol during magnetic resonance imaging. Journal of Biotechnology, 280, S35.

13. DORSCH, JA, DORSCH SE (2014). Understanding anesthesia equipment ( $\left.5^{\text {th }} \mathrm{edn}\right)$. Wolters Kluwer, India, pp. 298-300.

14. DRIEHUYS, B., NOULS, J., BADEA, A., BUCHOLZ, E., GHAGHADA, K., PETIET, A., \& HEDLUND, L.W. 
(2008). Small animal imaging with magnetic resonance microscopy. ILAR journal, 49(1), 35-53.

15. DUGDALE, A. (2010). Small animal sedation and premedication. Veterinary Anaesthesia Principle to Practice. West Sussex: Wiley-Blackwell, 30-44.

16. FIELDS, E.L., ROBERTSON, I.D., OSBORNE, J.A., \& BROWN JR, J.C. (2012). Comparison of abdominal computed tomography and abdominal ultrasound in sedated dogs. Veterinary Radiology \& Ultrasound, 53(5), 513-517

17. HALL, L.W., CLARKE, K.W., \& TRIM, C.M. (2001). Veterinary Anesthesia, Saunders.

18. HARRISON, R.L., CLARK, L., \& CORLETTO, F. (2012). Comparison of mean heart rate in anaesthetized dachshunds and other breeds of dog undergoing spinal Magnetic Resonance Imaging. Veterinary anaesthesia and analgesia, 39(3), 230-235.

19. JIMÉNEZ, C.P., MATHIS, A., MORA, S.S., BRODBELT, D., \& ALIBHAI, H. (2012). Evaluation of the quality of the recovery after administration of propofol or alfaxalone for induction of anaesthesia in dogs anaesthetized for magnetic resonance imaging. Veterinary anaesthesia and analgesia, 39(2), 151-159.

20. KHENISSI, L., COVEY-CRUMP, G., KNOWLES, T G., \& MURRELL, J. (2017). Do heat and moisture exchangers in the anaesthesia breathing circuit preserve body temperature in dogs undergoing anaesthesia for magnetic resonance imaging?. Veterinary anaesthesia and analgesia, 44(3), 452-460.

21. LEPPÄNEN, M.K., MCKUSICK, B.C., GRANHOLM, M.M., WESTERHOLM, F.C., TULAMO, R., \& SHORT, C.E. (2006). Clinical efficacy and safety of dexmedetomidine and buprenorphine, butorphanol or diazepam for canine hip radiography. Journal of small animal practice, 47(11), 663-669

22. LIAO, P., SINCLAIR, M., VALVERDE, A., MOSLEY, C., CHALMERS, H., MACKENZIE, S., \& HANNA, B. (2017). Induction dose and recovery quality of propofol and alfaxalone with or without midazolam coinduction followed by total intravenous anesthesia in dogs. Veterinary anaesthesia and analgesia, 44(5), 1016-1026.

23. LOVELL, D.P. (1986). Variation in pentobarbitone sleeping time in mice 1. Strain and sex differences. Laboratory animals, 20(2), 85-90.

24. LOZANO, A.J., BRODBELT, D.C., BORER, K.E., ARMITAGE-CHAN, E., CLARKE, K.W., \& ALIBHAI, H.I. (2009). A comparison of the duration and quality of recovery from isoflurane, sevoflurane and desflurane anaesthesia in dogs undergoing magnetic resonance imaging. Veterinary anaesthesia and analgesia, 36(3), 220-229.

25. MARTINEZ-TABOADA, F., \& LEECE, E.A. (2014). Comparison of propofol with ketofol, a propofolketamine admixture, for induction of anaesthesia in healthy dogs. Veterinary anaesthesia and analgesia, 41(6), 575-582.

26. MATHIS, A., PINELAS, R., BRODBELT, D.C., \& ALIBHAI, H.I. (2012). Comparison of quality of recovery from anaesthesia in cats induced with propofol or alfaxalone. Veterinary anaesthesia and analgesia, 39(3), 282-290.

27. MORADKHANI, M.R., KARIMI, A., NEGAHDARI, B., NADRI, S., \& EATEMADI, A. (2017). Drug anesthesia for children undergoing magnetic resonance imaging: A review. Biomedicine \& Pharmacotherapy, 88, 1183-1187.

28. NAGOSHI, M., REDDY, S., BELL, M., CRESENCIA, A., MARGOLIS, R., WETZEL, R., \& ROSS, P. (2018). Low-dose dexmedetomidine as an adjuvant to propofol infusion for children in MRI: A doublecohort study. Pediatric Anesthesia.

29. PICARD, V. (2000). Comparison of recovery of sevoflurane and propofol anesthesia in children. Acta Anesthesiol Scand, 44(3), 307-310.

30. POLLARD, R.E., \& PASCOE, P.J. (2008). Severe reaction to intravenous administration of an ionic iodinated contrast agent in two anesthetized dogs. Journal of the American Veterinary Medical Association, 233(2), 274-278.

31. POLLARD, R.E., PUCHALSKI, S.M., \& PASCOE, P.J. (2008). Hemodynamic and serum biochemical alterations associated with intravenous administration of three types of contrast media in anesthetized dogs. American journal of veterinary research, 69(10), 1268-1273.

32. RODRIGO-MOCHOLÍ, D., WILLEMS, A., SCHAUVLIEGE, S., \& BOSMANS, T. (2015). Cardiopulmonary arrest in a cat as a result of a suspected anaphylactic reaction to an intravenously administered iodinated contrast agent. Veterinary anaesthesia and analgesia, 42(5), 554-555.

33. SMITH, J.A. (2015). Patient and Anesthetist Safety Considerations for Laser and Radiographic Procedures and Magnetic Resonance Imaging. Veterinary Anesthesia and Analgesia: The Fifth Edition of Lumb and Jones, 1015-1026.

34. SOARE, M., \& VLĂGIOIU, C. (2012). Evaluation of clinical and laboratory investigation techniques of mammary gland tumors in the female dog: bibliographic study. Romanian Biotechnological Letters, 17(6), 7797.

35. TREMOLEDA, J.L., MACHOLL, S., \& SOSABOWSKI, J.K. (2018). Anesthesia and Monitoring of Animals During MRI Studies. In Preclinical MRI (pp. 423439). Humana Press, New York, NY.

36. TRIMBLE, T., BHALLA, R.J., \& LEECE, E.A. (2018). Comparison of sedation in dogs: methadone or butorphanol in combination with dexmedetomidine intravenously. Veterinary Anaesthesia and Analgesia.

37. TUDOR, R.G., DEGAN, A., COSTEA, R., \& PREDOI, G. (2018). Tears production evaluation during a protocol of anaesthesia for geriatric dogs... Revista Romana de Medicina Veterinara, 28(3), 25-28.

38. USHER, A.G., KEARNEY, R.A., \& TSUI, B.C. (2005). Propofol total intravenous anesthesia for MRI in children. Pediatric Anesthesia, 15(1), 23-28. 